\title{
Persona-Storyboard Fusion: A Hybrid Approach to Improving Design Artifacts
}

\author{
Michael Stewart ${ }^{1}$, Jennifer Francois ${ }^{1}$, Hongbo Zhang $^{2}$, D. Scott McCrickard ${ }^{1}$ \\ ${ }^{1}$ Center for Human-Computer Interaction, Virginia Tech, 2202 Kraft Dr., \\ Blacksburg, VA 24060, USA \\ ${ }^{2}$ Human Factors, Industrial and Systems Engineering, Virginia Tech, 250 Durham \\ Hall, Blacksburg, VA 24060, USA \\ \{tgm, jenniff, hbzhang, mccricks\}@vt.edu
}

\begin{abstract}
We propose and explore a novel method for fusing personas and storyboards in iteration by novice and expert designers. Personas have been shown to provide benefits to designers in understanding their users and keeping them in mind while designing. Storyboards have been shown to facilitate the communication of process between designers and users, and between designers and developers. Our process demonstrates the power and limitations of personas and storyboards, seeking to improve the design artifacts' ability to inform the analysis of a product, and the resulting design implications. We describe the application of our process in a product design exercise. Through our study we saw the fusion of personas and storyboards facilitate the teaching of design.
\end{abstract}

Keywords: Personas, Storyboards

\section{Introduction}

Teaching or practicing engineering design is challenging due to its complex, multivariate, and multilevel nature. Because of the complexity of the subject, novice designers (NDs) may not always acquire a working knowledge of every design technique. This can lead to confusion and even misuse of the design knowledge. Integration of experience with these design tools into the curriculum can enhance the ND's comprehension of design tools and promote a holistic view of design.

A persona is an abstract representation of users in the form of an archetype character, introduced by Cooper [1]. Personas can be used throughout the stages of design, from contextual data inquiry to prototyping, as a reminder of the target users. However, personas alone do not offer the capability for directly guiding the design.

The storyboard is usually a low-fidelity paper based prototype mostly used in early design stages. It helps the designer to visualize an idea about the process of the target application and communicate it to all project stakeholders. The targeted user can use the storyboard to understand how they can interact with the different aspects of the product. Storyboards can facilitate designers in achieving common ground. Additionally, they give the designer an opportunity for visual reflection, which consists of several phases: simulation, evaluation, and decision [2]. 
Using personas alone has not always addressed design expectations due to low adoption among design teams [3, 4]. Among design teams which used personas, their use was limited to individual designers' internal concepts or to facilitation of communication, which limits its effectiveness in design [5]. The isolated use of personas has not always been successful in design, which can be attributed to the unconvinced, insincere use of personas $[3,5]$. The integrated use of personas with other design techniques such as storyboarding is still not well documented.

In this research, we investigated how to integrate personas with storyboards. In exploring the fusion of these two design techniques, we ran a study with participants from a graduate class. Ten NDs and three expert designers (EDs) participated in the design of the interface for a Google Android app [6]. We found that the framework can help novice designers in brainstorming their design, help expert designers to enlarge the design workspace, and facilitates agreement between NDs and EDs. We address how the framework can be used in academic settings to facilitate teaching of design.

\section{Persona-Storyboard Fusion}

NDs have difficulty using multiple or even single design technique(s). EDs also have difficulty improving on novice designers' designs such as NDs' storyboards. EDs can improve the NDs' storyboard in terms of aesthetics, HCI design principles, and the satisfaction of general design goals. However, these improvements may not be sufficient for EDs to reach common ground with NDs. It is difficult for EDs to know NDs' design background and motivation. The inevitable conflicts that arise during ED and ND cooperation often leads to incompleteness or incorrectness of design and slow progress of both EDs and NDs.

The persona-storyboard fusion approach seeks to address these conflicts. Our approach consists of the following steps. First, the NDs create personas and storyboards. Then, with the help of the EDs, they evolve them together; with the NDs encouraged to continue this evolution with feedback from the EDs. The EDs independently rate the NDs' personas on applicable dimensions of interest. The NDs then take this feedback from the EDs and use it to help them improve their personas. Because EDs independently rate the personas, the NDs can see issues of contention in their personas reflected in inter-rater disagreement. With their evolved personas, the NDs can concurrently evolve their storyboards. They attempt to reflect the relevant facets of the persona in the storyboard. Next EDs will develop multiple possible enhancements to each of the NDs' storyboards. The NDs can then take this feedback and use it to focus their personas and scenarios to make clear why their design is best for their persona, or otherwise to improve their storyboard. The design iterations could continue until satisfaction. The framework is shown in Figure 1.

In this process, there are a few important considerations. First, it is vital that the storyboard include the relevant facets of the personas that the ND created. It is possible to do this through the storyboard's imagery and/or its descriptions. Next, 
both EDs and NDs should work toward converging on a direction that agrees with the design goals.

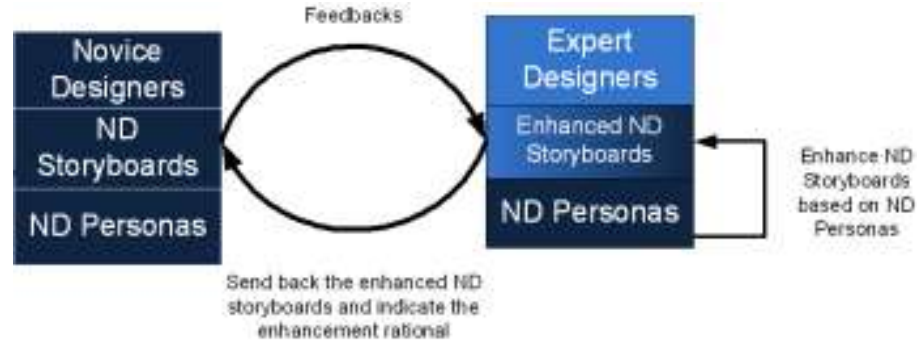

Figure 1. Storyboard and persona fusion diagram

The advantages of this framework are that it can give NDs further exposure to design techniques in action and that it brings the "living" nature of personas [7] to storyboarding. The possible disadvantages of this framework are that it may require a longer design cycle, and poor selection by NDs can possibly lead to biased design.

\section{Methods}

We applied our ND-ED method in the creation and evaluation of a mobile ideation tool that presented pictures of interesting uses of mobile technology to designers. The tool, a mobile version of our PIC-UP tool, supports flipping through lots of pictures and reading about the pictures, with the goal of conveying to designers the power and functionality of the Android mobile platform. The goal of the experiment was to come up with redesign ideas for the application. In total, 10 students taking a grad-level HCI class participated. These NDs had the opportunity to brainstorm their personas prior to class. They had to think about their background, interests, etc. During class, a presentation of personas and storyboards was given and a video of top 10 Android apps in 2010 was shown to stimulate the brainstorming for their storyboards. The NDs were then required to write down their persona information, and to create a storyboard for their idea for the redesign of PIC-UP.

The EDs then evaluated the personas from the NDs. Each ED evaluated the persona information independently and rated the persona information based on several categories from 0-4 (see Table 1). Based on these ratings, the EDs brainstormed independently how to improve the NDs' storyboards in ways that respect the ND's persona. By integrating the NDs personas, the EDs enhanced the storyboards and re-drew them using the Google Docs Drawing tool and finally, sent these revised storyboards back to the NDs. They were then asked to comment on the enhanced version of their storyboards. The EDs then modified the storyboards based on the NDs' feedback. After the enhancement, the EDs could find important features that could help with PIC-UP redesign. Then it was possible to consider these proposals and decide on the design tradeoffs of each before implementing them. 


\section{Results}

Many of the NDs were enthusiastic contributors to our study. However, there may have been some miscommunication in delivering our instructions as not all of them followed the instructions. This paper selects 3 individual submissions (labeled A, B, and $\mathrm{C}$ ) to examine the process. The ratings of seven categories of NDs' personas are shown in table 1. Each expert independently rated the personas on seven categories. The rating for each category ranges from 0 to 4 . In this table, the median and the individual ratings are shown. From Table 1, EDs have consistent view for several categories such as design experiences. EDs have diversified view of the ND's persona for some categories such as smart phone experiences.

Based on these ratings and the initial three storyboards created by the NDs, the EDs (in total 3) produced the enhanced storyboards for each of the NDs. In these 9 storyboards, the EDs ensured that the relevant facets of the persona were exemplified. This enabled the ND to see how other designers would interpret their storyboards, as well as their personas. For example, the ND may not have felt that the ED understood their persona fully. This could help indicate to the ND that they needed a more explicit description of that particular facet of their persona.

The EDs then sent these storyboards to the NDs and asked for their feedback. A few interesting points arose from the NDs' comments. ND A recognized the importance of the storyboard designer to be able to annotate elements of their storyboard with text. ND B recognized a tradeoff between the richness of the user experience in the Android App and the time available for development of the app. And ND C recognized the need for developers to be an audience to user feedback.

Table 1. Rating of seven categories extracted from personas (within cell, top: median of rating, bottom: individual rating) Experience (Exp), Development (Dev)

\begin{tabular}{|c|c|c|c|c|c|c|c|}
\hline ND & $\begin{array}{c}\text { Coding } \\
\text { Exp }\end{array}$ & $\begin{array}{c}\text { Design } \\
\text { Exp }\end{array}$ & $\begin{array}{c}\text { App dev } \\
\text { Exp }\end{array}$ & $\begin{array}{c}\text { Work } \\
\text { Exp }\end{array}$ & $\begin{array}{c}\text { Smart- } \\
\text { phone Exp }\end{array}$ & $\begin{array}{c}\text { Intro- } \\
\text { version }\end{array}$ & $\begin{array}{c}\text { Extro- } \\
\text { version }\end{array}$ \\
\hline \multirow{2}{*}{$\mathbf{A}$} & 2 & 3 & 0 & 0 & 1 & 2 & 1 \\
\cline { 2 - 8 } & $1,2,3$ & $3,4,3$ & $0,0,2$ & $0,0,1$ & $0,1,2$ & $0,2,4$ & $0,1,2$ \\
\hline \multirow{2}{*}{ B } & 2 & 4 & 0 & 0 & 3 & 3 & 0 \\
\cline { 2 - 8 } & $3,2,0$ & $4,4,4$ & $0,0,1$ & $0,0,1$ & $3,4,0$ & $3,0,4$ & $0,0,3$ \\
\hline \multirow{2}{*}{$\mathbf{C}$} & 1 & 3 & 2 & 2 & 2 & 3 & 1 \\
\cline { 2 - 8 } & $1,0,1$ & $2,3,4$ & $1,2,4$ & $4,2,2$ & $1,2,2$ & $4,0,3$ & $4,0,1$ \\
\hline
\end{tabular}

\section{Discussion and Conclusion}

Through our study we found three design implications for our framework. By quantitatively rating the NDs' personas, EDs have a firm basis to enhance the storyboards. Also, NDs and EDs have common ground based on the feedback process of personas and storyboards, which results in better communication and a design that reflects their common interests. As the final design incorporates features from the 
enhanced and critiqued storyboards, it effectively drew from a larger design space giving the designers more freedom.

We also experienced great variability in the quality of the personas. The primary issue was that not all of the NDs provided us with the dimensions for which we were hoping. We suggest that in the future, the EDs should be more explicit in communicating the primary descriptors they would like NDs to include in their personas. We also hoped that using the iterative process to inform the storyboards from the personas and to then have the personas be updated by the evolving storyboards would help in designing and enhancing personas as well as storyboards.

Not all of the NDs fully integrated their personas' facets in their storyboards. In order to help NDs include them, we recommend an example persona and storyboard with annotations that demonstrate details that reflect these facets. Additionally, in giving the feedback to the NDs after they create their own storyboards, the EDs should highlight aspects that reflect the personas. Then, to help the NDs enhance their personas, EDs could ask explicit questions about ambiguous aspects of the personas. This would help the NDs to see where they need to provide more detail in their personas. Finally, to help the NDs improve, it is important that the EDs provide detailed feedback in an accessible way. For example in our study, we should have shared our ratings of the personas with the NDs, aside from explicit questions, this would be an accessible way for the ND to see how EDs understand their personas and the level of ambiguity would be reflected in the inter-rater agreement.

Our hybrid approach in assisting novice and expert designers jointly participating in a fusion of two design techniques resulted in better communication between experts and novices, resulting in artifacts whose processes were arguably clearer and personas and storyboards which were arguably more directly relevant to stakeholders.

\section{References}

1. Cooper, A.: The inmates are running the asylum. Sams (1995)

2. Van der Lelie, C.: The value of storyboards in the product design process. Personal and Ubiquitous Computing 10, 159-162 (2006)

3. Blomquist, Å., Arvola, M.: Personas in action: Ethnography in an interaction design team. In: Conference Personas in action: Ethnography in an interaction design team. (Year)

4. Rowan, J., Cooper, M.: The plural self: Multiplicity in everyday life. Sage Publications Ltd (1999)

5. Ronkko, K.: An empirical study demonstrating how different design constraints, project organization and contexts limited the utility of personas. Proceedings of the 38th Annual Hawaii International Conference on System Sciences 220a-220a (2005)

6. Wahid, S., McCrickard, D.S., DeGol, J., Elias, N., Harrison, S.: Don't drop it! Pick it up and storyboard. Proceedings of the 29th international conference on Human factors in computing systems. (2011)

7. Nieters, J., Ivaturi, S., Ahmed, I.: Making Personas Memorable. Proceedings of The ACM CHI Conference on Human Factors in Computing Systems 1817-1824 (2007) 